\title{
SABR improves outcomes
}

According to the oligometastatic paradigm, patients with a limited number of metastatic lesions can be amenable to curative treatment if all lesions are resected or ablated using stereotactic ablative radiotherapy (SABR). Most of the evidence supporting this paradigm is from observational studies, but now the results of a randomized phase II trial indicate that SABR improves overall survival (OS) in these patients.

In the SABR-COMET trial,

99 patients with a controlled primary solid tumour and 1-5 metastatic lesions were randomly allocated to receive standard-of-care palliative treatment alone (control; $n=33$ ) or in addition to SABR delivered to all metastatic lesions $(n=66)$.

Median OS was longer with SABR than with no ablative treatment: 41 months versus 28 months (HR 0.57, 95\% CI 0.30-1.10; $P=0.09$ ). "We used a randomized phase II trial screening design, and thus a $P$ value $\leq 0.2$ for OS indicates a positive result," explains lead investigator David Palma.

Median progression-free survival was also longer with SABR: 12.0 months versus 6.0 months (HR 0.47, 95\% CI 0.30-0.76; $P=0.0012)$. The percentage of patients with control of all lesions that were present at randomization was $75 \%$ for patients receiving SABR versus $49 \%$ with no ablative treatment $(P=0.001)$. No significant differences were observed in quality-of-life scores (measured with the FACT-G tool).

The incidence of grade $\geq 2$ treatment-related adverse events (AEs) was higher with SABR: $29 \%$ versus $9 \%(P=0.026)$. Of note, 3 patients in the SABR group and none in the control group died from treatment-related toxicities.

In summary, evidence from a randomized trial now supports a survival benefit from SABR in oligometastatic disease. "We want

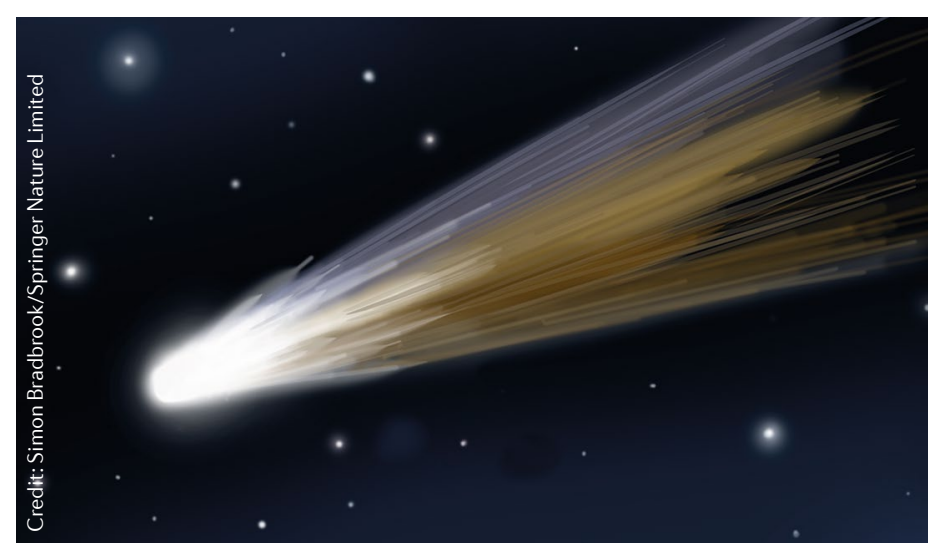

to prove this benefit in two follow-up phase III trials: the ongoing SABR-COMET-10 (NCT03721341), which involves patients with 4-10 metastatic lesions, and SABRCOMET-3 (NCT03862911), which is expected to open in 2-3 months and involves patients with 1-3 metastatic lesions," comments Palma.

Diana Romero

trial now

supports

a survival

benefit...

ORIGINAL ARTICLE Palma, D. A. et al.

Stereotactic ablative radiotherapy versus

standard of care palliative treatment in patients with oligometastatic cancers (SABR-COMET): a randomised, phase 2, open-label trial. Lancet https://doi.org/10.1016/S0140-6736(18)32487-5 (2019)

\section{GITR agonism combination is key}

Immunotherapy with agonistic antibodies to the glucocorticoidinduced tumour necrosis factor receptor-related protein (GITR) has shown promise in preclinical cancer models. Now, the results of a firstin-human study of one such agent, TRX518, have been reported.

In this phase I trial, 43 patients with advanced-stage solid tumours were treated with TRX518 at various doses. Pharmacodynamic studies revealed that TRX518 monotherapy effectively depleted regulatory $T\left(T_{\text {reg }}\right)$ cells from peripheral blood, interestingly, in apparent direct correlation with $\mathrm{T}_{\text {reg }}$ cell depletion in tumour specimens. This finding raises the possibility of a minimally invasive biomarker assay of the effect of GITR agonists in tumours. However, blood $\mathrm{CD}^{+}$or $\mathrm{CD} 4^{+}$effector $\mathrm{T}\left(\mathrm{T}_{\text {eff }}\right)$ cell and natural killer cell populations were not notably modulated and, disappointingly, no objective clinical responses were observed: the best response was stable disease $(n=4)$.

In keeping with these clinical findings, mice with established tumours were found to be resistant to GITR agonism despite robust depletion of intratumoural $\mathrm{T}_{\text {reg }}$ cells and corresponding increases in the ratio of $\mathrm{T}_{\text {eff }}$ cells to $\mathrm{T}_{\text {reg }}$ cells. By comparison, early tumours treated with a GITR agonist were more rapidly depleted of - and thus failed to accumulate $-T_{\text {reg }}$ cells and were responsive to treatment. $T_{\text {reg }}$ cell accumulation in established tumours before treatment was associated with imprinting of $\mathrm{T}_{\text {eff }}$ cells with an exhausted phenotype, which could not subsequently be reversed by $\mathrm{T}_{\text {reg }}$ cell depletion alone. Accordingly, exhausted, PD-1 ${ }^{+} \mathrm{T}$ cells persisted in patient tumour specimens with the highest levels of $\mathrm{T}_{\text {reg }}$ cell depletion. Importantly, however, reinvigoration of T cells via PD-1 inhibition rescued the responsiveness of established mouse tumours to GITR agonism.
"Studies of the pharmacodynamic effects of GITR agonism with TRX518 enabled us to design the appropriate treatment regimen for rational, science-based combination therapy," summarizes study co-lead Taha Merghoub. "We plan to further study the effects of GITR agonism in combination with PD-1 antagonism or chemotherapy and define biomarkers of clinical responses," he adds. Indeed, the authors have initiated a trial using TRX518 in combination with PD-1 inhibitors (NCT02628574), with promising clinical responses already observed, including after disease progression on prior anti-PD-1 therapy.

"Our study shows that conclusions on the importance of a pathway or drug should not be based solely on therapeutic outcomes: in depth study of the biological effects of a drug is key to designing efficacious treatment regimens," Merghoub concludes.

David Killock

ORIGINAL ARTICLE Zappasodi, R. et al. Rational design of anti-GITR-based combination immunotherapy. Nat. Med. https://doi.org/10.1038/ s41591-019-0420-8 (2019) 